\title{
Accretion physics of AM Herculis binaries
}

\section{Results from one-dimensional stationary radiation hydrodynamics}

\author{
A. Fischer ${ }^{\star}$ and K. Beuermann \\ Universitäts-Sternwarte, Geismarlandstr. 11, 37083 Göttingen, Germany \\ Received 30 November 2000 / Accepted 23 April 2001

\begin{abstract}
We have solved the one-dimensional stationary two-fluid hydrodynamic equations for post-shock flows on accreting magnetic white dwarfs simultaneous with the fully frequency and angle-dependent radiative transfer for cyclotron radiation and bremsstrahlung. Magnetic field strengths $B=10$ to $100 \mathrm{MG}$ are considered. At given $B$, this theory relates the properties of the emission region to a single physical parameter, the mass flow density (or accretion rate per unit area) $\dot{m}$. We present the normalized temperature profiles and fit formulae for the peak electron temperature, the geometrical shock height, and the column density of the post-shock flow. The results apply to pillbox-shaped emission regions. With a first-order temperature correction they can also be used for narrower columns provided they are not too tall.
\end{abstract}

Key words. stars: cataclysmic variables - stars: white dwarfs -stars: binaries: close - radiative transfer hydrodynamics

\section{Introduction}

The thermal structure of the accretion columns on accreting magnetic white dwarfs can be derived analytically for a single-particle fluid and sufficiently simple assumptions on the radiative cooling (Aizu 1973; Chevalier \& Imamura 1982; Wu et al. 1994). For the more general case of the optically thick frequency and angle-dependent radiative transfer in a two-fluid plasma, the coupled hydrodynamic and radiative transfer equations have to be solved numerically (Woelk \& Beuermann 1996, henceforth WB96). In this paper, we present results which are improved and expanded over those of WB96. We obtain the temperature and density profiles for plane-parallel post-shock cooling flows and derive fit formulae for the peak electron temperature $T_{\max }$, the column density $x_{\mathrm{s}}$, and the geometrical shock height $h_{\mathrm{sh}}$ as functions of the magnetic field strength $B$ and the mass flow density (accretion rate per unit area) $\dot{m}$. For low $\dot{m}$ and high $B$, we show that the shock solution merges into the non-hydrodynamic bombardment solution for an atmosphere which is heated by a stream of fast ions and cools by cyclotron radiation (Woelk \& Beuermann 1992, 1993, henceforth WB92, WB93).

Send offprint requests to: K. Beuermann,

e-mail: beuermann@uni-sw.gwdg. de

* Present address: Lufthansa Systems GmbH, 65451 Kelsterbach, Germany.
Our treatment of radiation-hydrodynamics is onedimensional and stationary. The one-dimensionality implies that our solutions are strictly applicable only to pillbox-shaped emision regions with a width $D \gg h_{\mathrm{sh}}$ and a stand-off distance $h_{\mathrm{sh}} \ll R_{\mathrm{wd}}$, where $R_{\mathrm{wd}}$ is the white dwarf radius. The stationarity implies that our solutions describe the mean properties of the shocks and that aspects like rapid fluctuations in the mass flow density and the stability against shock oscillations are left aside. Shock oscillations have been treated by a number of authors (Imamura et al. 1996; Saxton \& Wu 1999, and references therein) and generally suggest that cyclotron cooling stabilizes the flow and bremsstrahlung cooling destabilizes it. Observationally, optical oscillations have been found in a few polars, while the search for hard X-ray oscillations has so far yielded only upper limits (Larsson 1992; Wolff et al. 1999; Imamura et al. 2000, and references therein).

\section{Two-fluid radiation hydrodynamics}

\subsection{General approach}

We solve the stationary, one-dimensional, two-fluid hydrodynamic equations simultaneous with the frequency and angle-dependent radiative transfer, closely following the approach of WB96. We deviate from WB96 in the treatment of the shock itself. Instead of integrating the flow through the shock with an artificial viscosity, we adopt the presence of a strong ion shock and start the integration 
with adopted values of the ion and electron temperatures (see below). Of course, the solution now fails to reproduce the rapid rise in ion temperature across the shock, but otherwise the results are practically identical except for small differences at low velocities where the flow connects to the atmosphere of the star and large gradients revive the viscous terms again. The set of differential equations then reads (compare Eqs. (1) to (4), (7), and (8) of WB96)

$$
\begin{aligned}
& \rho v=-\dot{m} \\
& \rho v \frac{\mathrm{d} v}{\mathrm{~d} x}+\frac{\mathrm{d}}{\mathrm{d} x}\left(P_{\mathrm{i}}+P_{\mathrm{e}}\right)=-g \\
& \rho v \frac{\mathrm{d} \mathcal{E}_{\mathrm{i}}}{\mathrm{d} x}-v\left(P_{\mathrm{i}}+\mathcal{E}_{\mathrm{i}}\right) \frac{\mathrm{d} \rho}{\mathrm{d} x}=-\Lambda_{\mathrm{ei}} \\
& \rho v \frac{\mathrm{d} \mathcal{E}_{\mathrm{e}}}{\mathrm{d} x}-v\left(P_{\mathrm{e}}+\mathcal{E}_{\mathrm{e}}\right) \frac{\mathrm{d} \rho}{\mathrm{d} x}=\Lambda_{\mathrm{ei}}-\rho \frac{\mathrm{d} F_{\mathrm{rad}}}{\mathrm{d} x} \\
& \rho \cos \vartheta \frac{\mathrm{d} I_{\nu}(\vartheta)}{\mathrm{d} x}=\left[\kappa_{\nu}(\vartheta)+\sigma\right] I_{\nu}(\vartheta)-\kappa_{\nu}(\vartheta) B_{\nu}\left(T_{\mathrm{e}}\right)-\sigma J_{\nu}(5) \\
& F_{\mathrm{rad}}=2 \pi \int_{0}^{+1} \int_{-1}^{+1} I_{\nu}(\vartheta) \cos \vartheta \mathrm{d}(\cos \vartheta) \mathrm{d} \nu
\end{aligned}
$$

where $\rho$ is the mass density, $v$ the velocity, $\dot{m}$ the mass flow density, $P_{\mathrm{i}}$ and $P_{\mathrm{e}}$ the ion and electron pressures, $\mathcal{E}_{\mathrm{i}}$ and $\mathcal{E}_{\mathrm{e}}$ ion and electron internal energy densities, $I_{\nu}(\vartheta)$ the specific intensity of the radiation field at frequency $\nu$ and angle $\vartheta, J_{\nu}$ the angle-averaged intensity, $\kappa_{\nu}(\vartheta)$ the angledependent absorption coefficient, $\sigma$ the Thomson scattering coefficient, $B_{\nu}\left(T_{\mathrm{e}}\right)$ the Planck function at electron temperature $T_{\mathrm{e}}$, and $F_{\text {rad }}$ the total radial energy flux in the radiation field. We choose the downstream column density $x$ as the independent variable rather than the radial coordinate or the geometrical height $h$. Following WB96, we neglect the effects of radiation pressure and thermal conduction.

The connecting link between the hydrodynamics (Eqs. (1) to (4)) and the radiative transfer (Eqs. (5) and $(6))$ is $F_{\text {rad }}$ : the electron gas cools by radiation and is heated by Coulomb interactions with the ions, described by the non-relativistic electron ion energy exchange rate $\Lambda_{\text {ei }}$ (Spitzer 1956, see also WB96, their Eqs. (5) and (6)). The fully angle and frequency-dependent radiative transfer accounts for cyclotron absorption, free-free absorption, and coherent electron scattering. Our emphasis is on the largely correct treatment of the cyclotron spectra and we accept inaccuracies of the hard X-ray spectra caused by the neglect of Compton scattering. This still rather general treatment ensures that our results are relevant for a wide range of $\dot{m}$ including the low- $\dot{m}$ regime where radiative losses by optically thick cyclotron radiation dominate. The cyclotron absorption coefficients used here are the added coefficients for the ordinary and the extraordinary rays (WB92). This limitation is dropped in Sect. 3.1, below.

We use a Rybicki code for the LTE radiative transfer and integrate the set of equations implicitly, using a
Newton scheme to iterate between hydrodynamics and radiation transport. For more details see WB96. Our solution is strictly valid only for an infinite plane parallel layer. A first-order correction to the peak electron temperature for emission regions of finite lateral extent $D$ (Fig. 1) is discussed in Sects. 3.2 and 3.3 below.

Equation (2) accounts for post-shock acceleration and heating of the flow by the constant gravity term $g=$ $\mathrm{G} M_{\mathrm{wd}} / R_{\mathrm{wd}}{ }^{2}$. Within our one-dimensional approximation which disregards the convergence of the polar field lines, considering the variation of gravity with radius would not be appropriate. Our approach is, therefore, limited to stand-off distances of the shock $h_{\mathrm{sh}} \ll R_{\text {wd }}$. Settling solutions with $h_{\mathrm{sh}} \gtrsim R_{\mathrm{wd}}$ are not considered.

As in WB96, we assume that the pre-shock flow is fully ionized, but cold. Soft X-rays will photoionize the infalling matter and create a Strömgren region with a temperature typical of planetary nebulae, but for our purposes this is cold. Heating of the pre-shock electrons by thermal conduction may be more important. Equilibrium between diffusion and convection defines an electron precursor with a radial extent $\lambda_{\text {pre }} \simeq 4 \times 10^{-15} T_{\mathrm{e}, \mathrm{s}}^{5 / 2} / \dot{m} \mathrm{~cm}$, where $T_{\mathrm{e}, \mathrm{s}}$ is the electron temperature at the shock in $\mathrm{K}$ (Imamura et al. 1987) and $\dot{m}$ is in $\mathrm{g} \mathrm{cm}^{-2} \mathrm{~s}^{-1}$. Near the one-fluid limit, electron and ion shock temperatures are similar, $T_{\mathrm{e}, \mathrm{s}} \simeq T_{\mathrm{i}, \mathrm{s}}$, and the precursor extends to $\lambda_{\text {pre }} \simeq 0.09 h_{\mathrm{sh}}$. In a cyclotron-dominated plane-parallel flow, however, two effects cause the precursor to be less important: (i) the electrons never reach the peak temperature expected from one-fluid theory and (ii) the optically thick radiative transfer in the plane-parallel geometry sets up a radial temperature gradient which further depresses the electron temperature at the shock. In this paper, we do not consider thermal conduction, neglect the presence of the electron precursor, and opt to set $T_{\mathrm{e}, \mathrm{s}}=0$.

At $x=0$, we adopt the Rankine-Hugoniot jump conditions for a gas with adiabatic index $5 / 3$, i.e. we set the post-shock density to $4 \rho_{\mathrm{o}}$, the bulk velocity to $v_{\mathrm{o}} / 4$, and the pressure to $(3 / 4) \rho_{\mathrm{o}} v_{\mathrm{o}}^{2}$, with $\rho_{\mathrm{o}}$ and $v_{\mathrm{o}}$ the density and bulk velocity in the pre-shock flow. With $T_{\mathrm{e}, \mathrm{s}}=0$, the ion shock temperature is

$T_{\mathrm{i}, \mathrm{s}}=3 \mu_{\mathrm{i}} m_{\mathrm{u}} /(16 k) v_{\mathrm{o}}^{2}$.

We use $v_{\mathrm{o}}=\left(2 \mathrm{G} M_{\mathrm{wd}} / R_{\mathrm{wd}}\right)^{1 / 2}$ with Nauenberg's (1972) relation between mass and radius of the white dwarf. $\mu_{\mathrm{i}}$ is the molecular weight of the ions, $m_{\mathrm{u}}$ the mass unit, and $k$ the Boltzmann constant.

All numerical calculations are performed for a hydrogen plasma with $\mu=0.5$ and $\mu_{\mathrm{i}}=\mu_{\mathrm{e}}=\mu_{Z}=1$, where $\mu_{\mathrm{e}}$ is the number of nucleons per electron, $\mu$ is the molecular weight of all particles, and $\mu_{Z}$ the molecular weight of the ions weighted with $Z_{\mathrm{k}}^{2}$. We include the molecular weight dependence in our equations in order to allow conversion to other compositions, e.g., a fully ionized plasma of solar composition with $\mu=0.617, \mu_{\mathrm{i}}=1.297, \mu_{\mathrm{e}}=1.176$, and $\mu_{Z}=0.927$. 


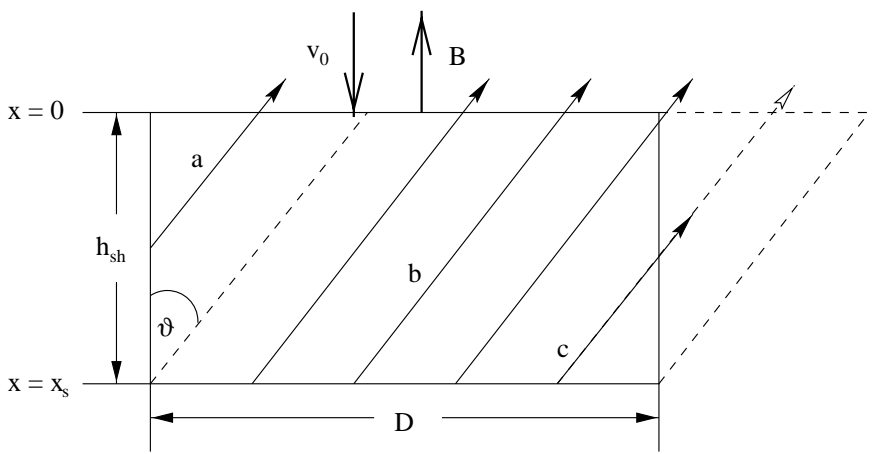

Fig. 1. Schematics of the emission region. The region is bounded at the top by the shock front and at the bottom by the white dwarf.

\subsection{Bremsstrahlung and cyclotron emissivities}

The frequency-integrated volume emissivity for bremsstrahlung is

$\epsilon_{\text {brems }}=c_{2} T^{1 / 2} n_{\mathrm{e}} \sum_{k} n_{k} Z_{k}^{2}=\frac{c_{2}}{\left(k m_{\mathrm{u}}^{3}\right)^{1 / 2}} \frac{\mu^{1 / 2}}{\mu_{\mathrm{e}} \mu_{Z}} P^{1 / 2} \rho^{3 / 2}(8)$

where $c_{2}=1.43 \times 10^{-27}$ cgs-units, $n_{\mathrm{e}}$ is the number density of the electrons, $n_{k}$ the number density of the ions of charge $Z_{k} \mathrm{e}, P$ is the gas pressure, and $\rho$ the mass density. The Thomson scattering optical depth of a bremsstrahlung dominated flow parallel to the flow is of order unity, implying that bremsstrahlung is essentially optically thin (Aizu 1973).

We use the cyclotron absorption coefficients for the ordinary and extraordinary rays given by Chanmugam \& Dulk (1981), Thompson \& Cawthorne (1987), and WB92. The total cyclotron emissivity of non-relativistic electrons integrated over wavelength and solid angle is

$\epsilon_{\mathrm{cyc}} \propto n_{\mathrm{e}} T B^{2} \propto\left(\mu / \mu_{\mathrm{e}}\right) B^{2} P$.

In the columns considered, cyclotron radiation is optically thin in the higher harmonics, but is always optically thick in the first few harmonics, and the temperature distribution in a cyclotron-dominated emission region can properly be calculated only by solving the coupled radiationhydrodynamic equations.

\subsection{Geometry of the emission region}

Figure 1 shows the schematic of an emission region with finite lateral extent $D$. The shock is located at $h=h_{\mathrm{sh}}$ above the white dwarf surface. The downstream column density is $x=0$ at the shock and and $x=x_{\mathrm{s}}$ at the surface of the star, with $x$ and $h$ being related by $\mathrm{d} x=-\rho \mathrm{d} h$. The gravity vector $\boldsymbol{g}$ and the magnetic field vector $\boldsymbol{B}$ are taken parallel to the flow lines. The radiation-hydrodynamic equations are solved for layers of infinite $D$ to yield the run of electron temperature and mass density, $T_{\mathrm{e}}(x)$ and $\rho(x)$. These profiles are later employed to calculate the outgoing spectra for emission regions with finite $D$ by ray tracing, i.e. by adding the contributions from an appropriate number of rays (Fig. 1 and Sect. 3.1). This procedure is not self-consistent if optically thick radiative losses occur from the sides of the column. An appropriate first-order correction to the temperature structure derived for the infinite layer is discussed in Sects. 3.2 and 3.3, below. The treatment of really tall columns requires a different approach which specifically allows for the emission from the sides of the column (Wu et al. 1994).

Radiation intercepted by the white dwarf is either reflected or absorbed and reemitted by its locally heated atmosphere. We assume coherent scattering of hard X-rays using the frequency-dependent reflection albedo $A_{\nu}$ of van Teeseling et al. (1994). The fraction $1-A_{\nu}$ of the energy is re-emitted in the UV and soft X-ray regime and is not considered in this paper.

\subsection{Limiting cases}

Here, we consider simple limiting cases which can, in part, be solved analytically. Below, we shall discuss our numerical results in terms of these limiting solutions. The high $\dot{m}$, low $B$ limit is the bremsstrahlung-dominated one-fluid solution. In the opposite limit of low $\dot{m}$, high $B$ one enters the non-hydrodynamic regime (Lamb \& Masters 1977). Here, the bombardment solution of a static atmosphere heated by a stream of fast ions and cooling by cyclotron emission is an appropriate approximation (Kuijpers \& Pringle 1982, WB92, WB93).

The one-dimensional, one-fluid hydrodynamic equations with simple terms for optically thin cooling can be solved analytically (Aizu 1973; Chevalier \& Imamura 1982). Integration of Eq. (2) with Eq. (1), $P=P_{\mathrm{i}}+P_{\mathrm{e}}$, and $g=0$ yields $P=\dot{m}\left(v_{\mathrm{o}}-v\right)$ which allows to express the emissivities of Sect. 2.2 as $\epsilon_{\text {brems }} \propto \dot{m}^{2} f(v)$ and $\epsilon_{\text {cyc }} \propto \dot{m} \mathrm{~g}(v)$, with $f$ and $g$ being functions of the flow velocity $v$ and with additional dependencies on the $\mu$ 's and $B$ contained in the proportionality factors. Integration of the energy equation over $v$ yields expressions for the column density $x_{\mathrm{s}}$ and the geometrical shock height $h_{\mathrm{sh}}$ which reflect the parameter dependence of $\epsilon$,

$$
\begin{array}{r}
x_{\mathrm{s}} \propto \frac{\dot{m}^{2}}{\epsilon} \begin{cases}x_{\mathrm{s}, \mathrm{brems}}=\text { constant } & \text { for } \epsilon=\epsilon_{\mathrm{brems}} \\
x_{\mathrm{s}, \mathrm{cyc}} \propto \dot{m} & \text { for } \epsilon=\epsilon_{\mathrm{cyc}},\end{cases} \\
h_{\mathrm{sh}} \propto \frac{\dot{m}}{\epsilon} \begin{cases}h_{\mathrm{sh}, \mathrm{brems}}=1 / \dot{m} & \text { for } \epsilon=\epsilon_{\mathrm{brems}} \\
h_{\mathrm{sh}, \mathrm{cyc}} \propto \text { constant } & \text { for } \epsilon=\epsilon_{\mathrm{cyc} .}\end{cases}
\end{array}
$$

In the flows considered here, bremsstrahlung is close to optically thin and the analytical solution is quantitatively corroborated by our numerical results. Cyclotron emission, on the other hand, is optically thick in the lower harmonics which reduces the effective emissivity and inflates the emission region. While the $\dot{m}$-dependence of cyclotrondominated columns in Eqs. (10) and (11) is recovered in our numerical calculations, not surprisingly, the numerically derived sizes of $x_{\mathrm{s}}$ and $h_{\mathrm{sh}}$ are much larger than those predicted by the (unquoted) proportionality factors for cyclotron cooling in Eqs. (10) and (11). 


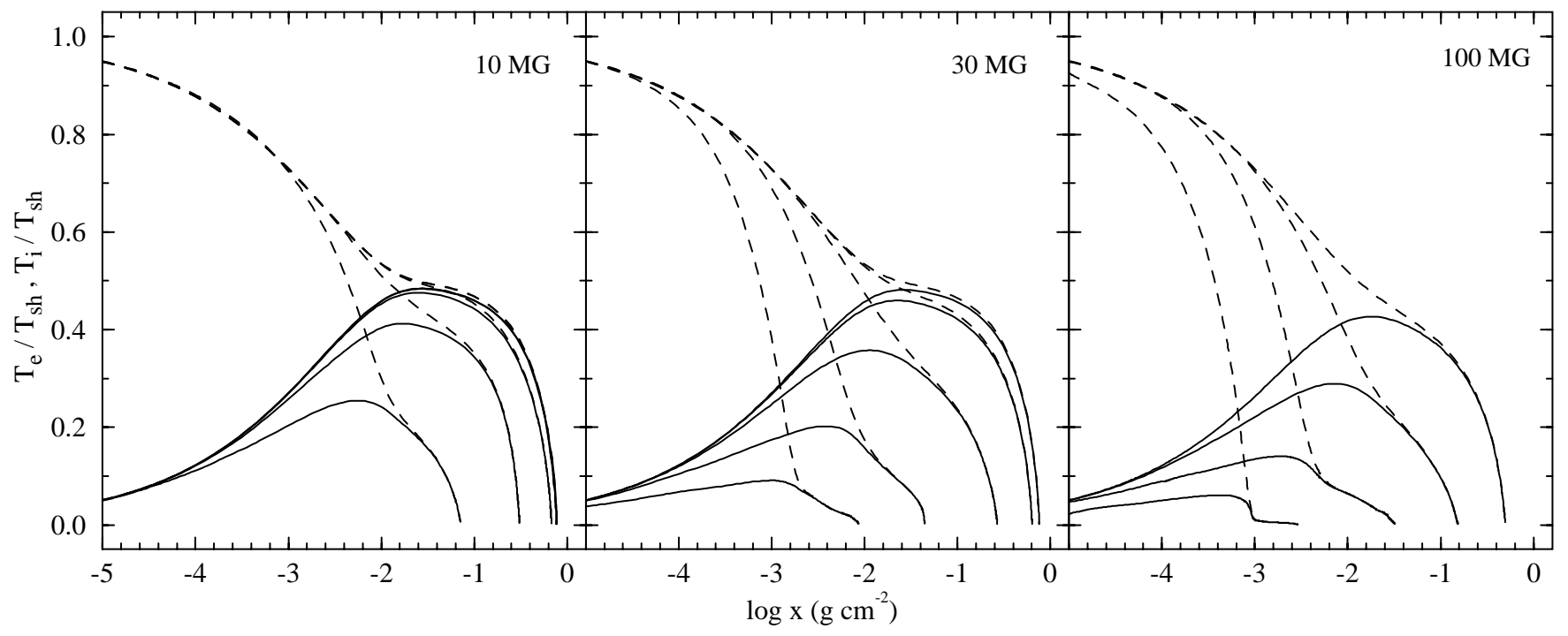

Fig. 2. Temperature profiles for the ions (dashed curves) and electrons (solid curves) as functions of column density $x$ for $M_{\mathrm{wd}}=0.6 M_{\odot}$ and field strengths of $10 \mathrm{MG}$ (left panel), $30 \mathrm{MG}$ (center panel) and $100 \mathrm{MG}$ (right panel). The individual curves are for mass flow densities $\dot{m}=100,10,1,10^{-1}$ and $10^{-2} \mathrm{~g} \mathrm{~cm}^{-2} \mathrm{~s}^{-1}$ (from top). In the left panel, the curves for 100 and $10 \mathrm{~g} \mathrm{~cm}^{-2} \mathrm{~s}^{-1}$ are indistinguishable. In the right panel, the bottom curve is for $10^{-1} \mathrm{~g} \mathrm{~cm}^{-2} \mathrm{~s}^{-1}$. From Eqs. (13) and (16), $x_{\mathrm{s}, \mathrm{brems}}=0.783 \mathrm{~g} \mathrm{~cm}^{-2}\left(\log x_{\mathrm{s}, \mathrm{brems}}=-0.106\right)$ and $T_{\max , \text { brems }}=T_{\mathrm{i}, \mathrm{s}} / 2$.

\subsection{Bremsstrahlung-dominated shock solution}

For a strong shock in a one-fluid plasma with adiabatic index $5 / 3$, the normalized post-shock velocity $\omega=4 v / v_{\mathrm{o}}$ varies between 1 and 0 . The column density $x$ measured from the shock is related to $\omega$ by (Aizu 1973; Chevalier \& Imamura 1982)

$x=c_{1} v_{\mathrm{o}}^{2}\left[\sqrt{3}-\frac{\pi}{3}-\frac{1+\omega}{2} \sqrt{4 \omega-\omega^{2}}+\cos ^{-1}\left(1-\frac{\omega}{2}\right)\right]$.

The total column density and shock height are given by

$$
\begin{aligned}
x_{\mathrm{s}, \text { brems }} & =c_{1} v_{\mathrm{o}}^{2}(\sqrt{3}-\pi / 3) \mathrm{g} \mathrm{cm}^{-2} \\
h_{\mathrm{sh}, \text { brems }} & =c_{1} v_{\mathrm{o}}^{3}(39 \sqrt{3}-20 \pi) /(48 \dot{m}) \quad \mathrm{cm},
\end{aligned}
$$

where $c_{1}=\left(k^{1 / 2} m_{\mathrm{u}}^{3 / 2} / 4 c_{2}\right)\left(\mu_{\mathrm{e}} \mu_{Z} / \mu^{1 / 2}\right)$ with values of $6.22 \times 10^{-18} \mathrm{cgs}$ for pure hydrogen and $6.10 \times 10^{-18} \mathrm{cgs}$ for solar composition. The temperature profile follows from pressure equilibrium $P=\rho v\left(v_{\mathrm{o}}-v\right)$, the equation of state for the ideal gas, and Eq. (1) as

$T=\frac{1}{3}\left(4 \omega-\omega^{2}\right) T_{\max , \text { brems }}$

with

$T_{\max , \text { brems }}=3 \mu m_{\mathrm{u}} /(16 k) v_{\mathrm{o}}^{2}=\left(\mu / \mu_{\mathrm{i}}\right) T_{\mathrm{i}, \mathrm{s}}$.

Our two-fluid calculations for high $\dot{m}$, low $B$ reproduce the temperature profile $T(x)$ given by Eq. (15) with (12) and (16) to better than $1 \%$ of $T_{\max }$,brems, except for the initial equilibration layer which is infinitely thin in the analytic calculation and has a finite thickness with rising electron temperature in our calculations. As an aside, we note that $T / T_{\max , \text { brems }} \simeq\left(1-x / x_{\mathrm{s}}\right)^{0.59}$ with an rms error of less than $1 \%$.

\subsection{Cyclotron-dominated bombardment solution}

The bombardment solution involves by nature a two-fluid approach. WB92 solved this case using a Fokker-Planck formalism to calculate the stopping length of the ions and a Feautrier code for the radiative transfer. WB93 (their Eqs. (8), (9)) provided power law fits to their numerical results for the column density and the peak electron temperature. Since the ions are slowed down by collisions with atmospheric electrons, a factor $\mu_{\mathrm{e}}$ appears in $x_{\mathrm{s}}$ :

$x_{\mathrm{s}, \text { bomb }}=3.94 \times 10^{-2} \mu_{\mathrm{e}}\left(\dot{m} B_{7}^{-2.6}\right)^{0.30} M_{\mathrm{wd}}^{1.72} \mathrm{~g} \mathrm{~cm}^{-2}$

$T_{\text {max }, \text { bomb }}=1.28 \times 10^{9}\left(\dot{m} B_{7}^{-2.6}\right)^{0.42} M_{\mathrm{wd}}^{0.66} \quad \mathrm{~K}$.

Here, $\dot{m}$ is in $\mathrm{g} \mathrm{cm}^{-2} \mathrm{~s}^{-1}, B_{7}$ is in units of $10^{7} \mathrm{G}$, and $M_{\mathrm{wd}}$ is in solar masses. These fits are very close to the quasianalytical expressions of Eqs. (5) and (6) of WB93 ${ }^{1}$.

With increasing $\dot{m}$, a shock develops which is initially cyclotron-dominated and ultimately bremsstrahlungdominated. Since $T_{\max }$,bomb reaches $T_{\max }$,brems at some intermediate $\dot{m}$, we expect a smooth transition in peak temperature between these cases. The situation is quite different for $x_{\mathrm{s}}$, however. At the $\dot{m}$ where $T_{\max }$ bomb equals $T_{\text {max,brems }}, x_{\mathrm{s}, \text { bomb }}$ and $x_{\mathrm{s} \text {, brems }}$ differ by more than two orders of magnitude. The run of $x_{\mathrm{s}}(\dot{m})$ between these two limiting cases can be determined only with a radiationhydrodynamical approach.

The bombardment solution does not predict the geometrical scale height of the heated atmosphere which we expect to lie between that of a corona with an external pressure $P=0$ and that of a layer compressed by the ram pressure $P=\rho_{\mathrm{o}} v_{\mathrm{o}}{ }^{2}$.

${ }^{1}$ Note the misprint in Eq. (6) of WB93 which should read $B^{0.85}$ instead of $B^{1.85}$. 


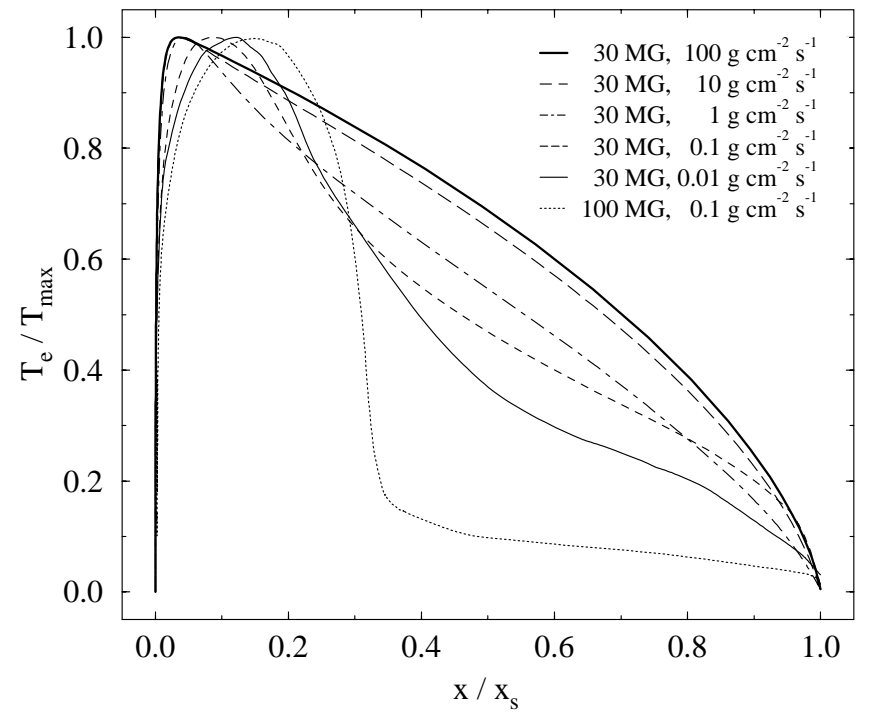

Fig. 3. Normalized electron temperature distributions for $M_{\mathrm{wd}}=0.6 \quad M_{\odot}$ and the values of $B$ and $\dot{m}$ given in the figure.

\subsection{Parameterization of the results}

In the bombardment solution (Eqs. (17), (18)), the dependence of $x_{\mathrm{s}}$ and $T_{\max }$ on $\dot{m} B^{-2.6}$ is obtained from the equilibrium between the energy gain by accretion, $F_{\text {acc }} \propto \dot{m}$, and the energy loss by optically thick cyclotron radiation, $F_{\text {cyc }} \propto T_{\max } \omega_{*}^{3}$, where $\omega_{*}=m_{*} \omega_{\mathrm{c}}$ is the high-frequency cutoff of the cyclotron spectrum and $\omega_{\mathrm{c}} \propto B$ the cyclotron frequency. We determine the limiting harmonic number $m_{*}$ from the cyclotron calculations of Chanmugam \& Langer (1991; their Fig. 5) as $m_{*}=4.43 \Lambda_{4}^{a} T_{8}^{b}$ with $a \simeq 0.12$ and $b \simeq 0.40$. This approximation is valid near depth parameters $\Lambda_{4}=\Lambda / 10^{4} \simeq 1$ and temperatures $T_{8}=T_{\max } /\left(10^{8} \mathrm{~K}\right) \simeq 1$ and is more adequate for the cyclotron-dominated emission regions on polars than the frequently quoted formula of Wada et al. (1980). Replacing $\omega_{*}$ with $\Lambda \propto x_{\mathrm{s}} / B$ in $F_{\text {cyc }}$ and equating the accretion and radiative energy fluxes yields the result that a power of $T_{\max }$ is proportional to $\dot{m} B^{3(a-1)} \simeq \dot{m} B^{-2.6}$. The same holds for $x_{\mathrm{s}}$.

We find that the cyclotron-dominated shocks at low $\dot{m}$ behave similarly to bombarded atmospheres in that their thermal properties, too, depend on $\dot{m} B^{-2.6}$. The individual temperature profiles $T_{\mathrm{e}}(x)$ for different $\dot{m}, B$ with the same $\dot{m} B^{-2.6}$ coincide only in an approximate way, but the dependency on $\dot{m} B^{-2.6}$ holds quite well for the two characteristic values of each profile, $T_{\max }$ and $x_{\mathrm{s}}$. If we leave the exponent $\alpha$ in $\dot{m} B^{\alpha}$ as a fit variable, the smallest scatter in $T_{\max }$ and $x_{\mathrm{s}}$ as functions of $\dot{m} B^{\alpha}$ is, in fact, obtained for $\alpha=-2.6 \pm 0.2$.

Our model calculations cover magnetic field strengths $B=10-100 \mathrm{MG}$ and mass flow densities $\dot{m}=$ $10^{-2}-10^{2} \mathrm{~g} \mathrm{~cm}^{-2} \mathrm{~s}^{-1}$. In what follows, we present first the temperature and density profiles along the flow lines. From these, we obtain $T_{\max }, x_{\mathrm{s}}$, and $h_{\mathrm{sh}}$ as the

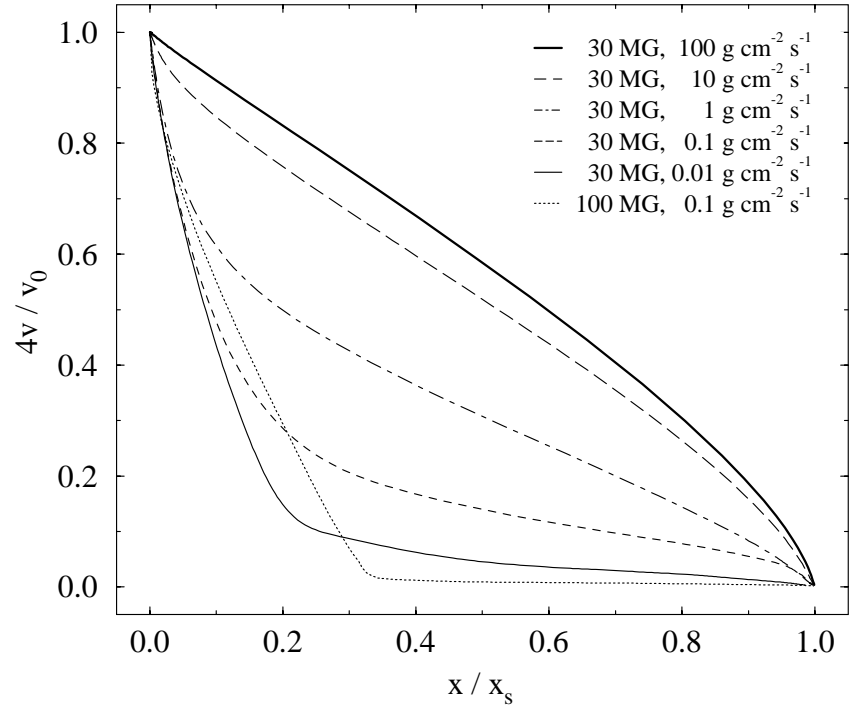

Fig. 4. Normalized velocity $w=4 v / v_{\mathrm{o}}$ for $M_{\mathrm{wd}}=0.6 M_{\odot}$ and the same values of field strength and mass flow density $\dot{m}$ as in Fig. 3.

characteristic parameters of the post-shock flow which are presented in an appropriate way as functions of $\dot{m} B^{-2.6}$.

\subsection{Electron temperature profiles for an infinite layer}

Figure 2 shows the temperature profiles $T_{\mathrm{i}}(x)$ and $T_{\mathrm{e}}(x)$ for $M_{\text {wd }}=0.6 M_{\odot}$ and several $\dot{m}-B$ combinations on a logarithmic depth scale which emphasizes the initial rise of the profiles. These profiles display a substantial spread in $x_{\mathrm{s}}$ and in $T_{\max }$, reflecting the influence of cyclotron cooling. At $10^{-2} \mathrm{~g} \mathrm{~cm}^{-2} \mathrm{~s}^{-1}, 100 \mathrm{MG}$, cyclotron cooling has reduced $T_{\max }$ to $6 \%$ and $x_{\mathrm{s}}$ to $0.3 \%$ of the respective values for the pure bremsstrahlung solution. We have confidence in our numerical results because they accurately reproduce the analytic bremsstrahlung solution (see above).

Figure 3 displays the normalized profiles of the electron temperature, $T_{\mathrm{e}} / T_{\max }$ vs. $x / x_{\mathrm{s}}$, for different $\dot{m}, B$ combinations, covering the range from a bremsstrahlungdominated flow with $100 \mathrm{~g} \mathrm{~cm}^{-2} \mathrm{~s}^{-1}$, 30MG (fat solid curve) to $10^{-2} \mathrm{~g} \mathrm{~cm}^{-2} \mathrm{~s}^{-1}, 100 \mathrm{MG}$ near the nonhydrodynamic limit (dotted curce). They represent an approximate sequence in $\dot{m} B^{-2.6}$, but not surprisingly, the shapes differ somewhat for different $\dot{m}$ and $B$ combinations with the same value of $\dot{m} B^{-2.6}$ (not shown in Fig. 3).

Equilibration between electron and ion temperatures is reached at column densities of $\sim 10^{-3} \ldots 10^{-1} \mathrm{~g} \mathrm{~cm}^{-2} \mathrm{~s}^{-1}$ depending on $\dot{m}$ and $B$ (Fig. 2). At $100 \mathrm{~g} \mathrm{~cm}^{-2} \mathrm{~s}^{-1}, 10 \mathrm{MG}$, electrons and ions equilibrate as early as $\sim 0.03 x_{\mathrm{s}}$, while at $10^{-2} \mathrm{~g} \mathrm{~cm}^{-2} \mathrm{~s}^{-1}, 100 \mathrm{MG}$, equilibration length and $x_{\mathrm{s}}$ are of the same order, indicating the approach to the non-hydrodynamic regime. A peculiar feature of the latter profile is the extended low-temperature tail which was not adequately resolved by WB96. This tail appears when equilibration occurs near the temperature at which cyclotron cooling becomes ineffective and the density is 
Table 1. (a) Top: temperature profiles $T_{\mathrm{e}} / T_{\max }\left(x / x_{\mathrm{s}}\right)$ for a white dwarf mass of $0.6 M_{\odot}$, field strengths of 10,30 , and $100 \mathrm{MG}$, and mass flow densities between 100 and $0.01 \mathrm{~g} \mathrm{~cm}^{-2} \mathrm{~s}^{-1}$. First line: analytical solution of Eqs. (12) and (15). Subsequent lines: profiles for parameter combinations $B, \dot{m}$ in $\mathrm{MG}$ and $\mathrm{g} \mathrm{cm}^{-2} \mathrm{~s}^{-1}$, with $B_{7}$ in units of $10^{7} \mathrm{G}$. All profiles start at $\left(x / x_{\mathrm{s}}, T_{\mathrm{e}} / T_{\max }\right)=(0,0)$, end at $(1,0)$, and are normalized to a peak value of unity. (b) Bottom: same for the normalized velocity $w=4 v / v_{\mathrm{o}}$. These profiles start at $\left(x / x_{\mathrm{s}}, w\right)=(0,1)$ and end at $(1,0)$.

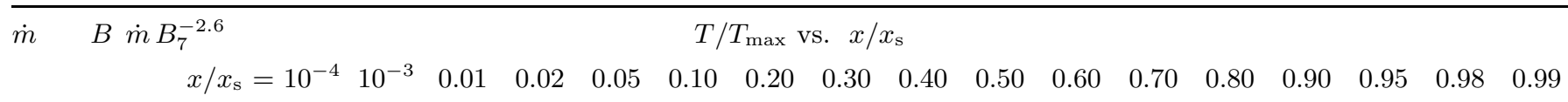

\begin{tabular}{llllllllllllllllllllllllll}
\hline & $\infty$ & 1.000 & 0.999 & 0.995 & 0.989 & 0.973 & 0.945 & 0.886 & 0.821 & 0.751 & 0.674 & 0.589 & 0.494 & 0.382 & 0.245 & 0.156 & 0.085 & 0.054 \\
100 & 10 & 100 & 0.230 & 0.515 & 0.916 & 0.984 & 0.995 & 0.967 & 0.905 & 0.840 & 0.770 & 0.691 & 0.603 & 0.505 & 0.392 & 0.250 & 0.158 & 0.085 & 0.053 \\
10 & 10 & 10 & 0.230 & 0.515 & 0.915 & 0.984 & 0.996 & 0.968 & 0.906 & 0.842 & 0.770 & 0.692 & 0.604 & 0.506 & 0.393 & 0.250 & 0.159 & 0.082 & 0.048 \\
1 & 10 & 1 & 0.219 & 0.499 & 0.898 & 0.977 & 0.998 & 0.973 & 0.914 & 0.850 & 0.782 & 0.710 & 0.629 & 0.536 & 0.426 & 0.281 & 0.176 & 0.085 & 0.047 \\
0.1 & 10 & 0.1 & 0.191 & 0.434 & 0.822 & 0.927 & 0.999 & 0.980 & 0.916 & 0.849 & 0.779 & 0.706 & 0.622 & 0.522 & 0.400 & 0.239 & 0.127 & 0.047 & 0.022 \\
0.01 & 10 & 0.01 & 0.175 & 0.395 & 0.743 & 0.861 & 0.981 & 0.991 & 0.860 & 0.743 & 0.650 & 0.565 & 0.476 & 0.384 & 0.285 & 0.165 & 0.092 & 0.039 & 0.021 \\
100 & 30 & 5.75 & 0.228 & 0.512 & 0.914 & 0.984 & 0.994 & 0.966 & 0.903 & 0.836 & 0.766 & 0.686 & 0.598 & 0.499 & 0.388 & 0.247 & 0.153 & 0.080 & 0.046 \\
10 & 30 & 0.58 & 0.224 & 0.504 & 0.909 & 0.981 & 0.994 & 0.958 & 0.888 & 0.813 & 0.738 & 0.657 & 0.569 & 0.473 & 0.361 & 0.225 & 0.134 & 0.065 & 0.035 \\
1 & 30 & 0.058 & 0.209 & 0.468 & 0.865 & 0.958 & 0.997 & 0.937 & 0.815 & 0.717 & 0.629 & 0.546 & 0.460 & 0.372 & 0.272 & 0.162 & 0.091 & 0.042 & 0.025 \\
0.1 & 30 & 0.0058 & 0.185 & 0.408 & 0.738 & 0.849 & 0.971 & 0.997 & 0.833 & 0.657 & 0.548 & 0.468 & 0.401 & 0.336 & 0.274 & 0.197 & 0.145 & 0.085 & 0.045 \\
0.01 & 30 & 0.00058 & 0.178 & 0.390 & 0.710 & 0.802 & 0.922 & 0.994 & 0.878 & 0.652 & 0.484 & 0.362 & 0.292 & 0.245 & 0.196 & 0.119 & 0.074 & 0.038 & 0.022 \\
100 & 100 & 0.25 & 0.222 & 0.497 & 0.898 & 0.979 & 0.992 & 0.942 & 0.849 & 0.766 & 0.685 & 0.603 & 0.517 & 0.424 & 0.317 & 0.188 & 0.104 & 0.044 & 0.020 \\
10 & 100 & 0.025 & 0.206 & 0.468 & 0.839 & 0.940 & 0.999 & 0.927 & 0.756 & 0.639 & 0.541 & 0.450 & 0.367 & 0.289 & 0.201 & 0.103 & 0.043 & 0.011 & 0.006 \\
1 & 100 & 0.0025 & 0.199 & 0.440 & 0.795 & 0.903 & 0.997 & 0.921 & 0.564 & 0.458 & 0.375 & 0.302 & 0.237 & 0.193 & 0.129 & 0.058 & 0.027 & 0.013 & 0.007 \\
0.1 & 100 & 0.00025 & 0.180 & 0.354 & 0.626 & 0.727 & 0.880 & 0.976 & 0.966 & 0.600 & 0.131 & 0.097 & 0.084 & 0.077 & 0.063 & 0.047 & 0.038 & 0.030 & 0.022 \\
\hline
\end{tabular}

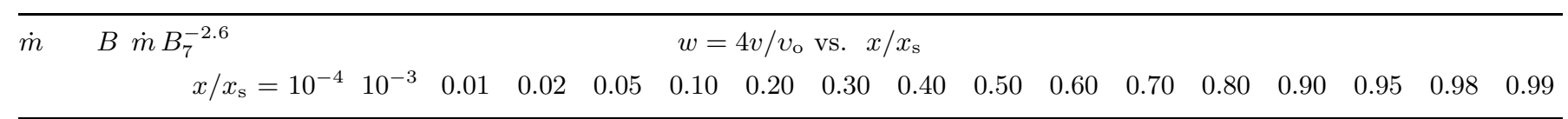

\begin{tabular}{lllllllllllllllllllllll}
\hline & & $\infty$ & 1.000 & 0.999 & 0.992 & 0.984 & 0.960 & 0.921 & 0.841 & 0.761 & 0.678 & 0.594 & 0.506 & 0.413 & 0.311 & 0.193 & 0.121 & 0.065 & 0.041 \\
100 & 10 & 100 & 1.000 & 0.999 & 0.992 & 0.985 & 0.961 & 0.920 & 0.840 & 0.760 & 0.676 & 0.593 & 0.504 & 0.411 & 0.310 & 0.191 & 0.119 & 0.064 & 0.039 \\
10 & 10 & 10 & 1.000 & 0.999 & 0.991 & 0.983 & 0.958 & 0.915 & 0.834 & 0.755 & 0.670 & 0.586 & 0.496 & 0.405 & 0.304 & 0.187 & 0.115 & 0.060 & 0.035 \\
1 & 10 & 1 & 1.000 & 0.998 & 0.983 & 0.969 & 0.932 & 0.882 & 0.788 & 0.700 & 0.618 & 0.538 & 0.459 & 0.376 & 0.289 & 0.182 & 0.112 & 0.053 & 0.029 \\
0.1 & 10 & 0.1 & 1.000 & 0.993 & 0.934 & 0.888 & 0.794 & 0.696 & 0.571 & 0.480 & 0.404 & 0.336 & 0.279 & 0.218 & 0.158 & 0.089 & 0.046 & 0.017 & 0.008 \\
0.01 & 10 & 0.01 & 1.000 & 0.989 & 0.892 & 0.811 & 0.635 & 0.440 & 0.266 & 0.193 & 0.150 & 0.116 & 0.090 & 0.066 & 0.045 & 0.024 & 0.013 & 0.005 & 0.003 \\
100 & 30 & 5.75 & 1.000 & 0.999 & 0.990 & 0.981 & 0.953 & 0.913 & 0.831 & 0.751 & 0.667 & 0.584 & 0.497 & 0.402 & 0.303 & 0.186 & 0.115 & 0.059 & 0.033 \\
10 & 30 & 0.58 & 1.000 & 0.996 & 0.972 & 0.948 & 0.902 & 0.947 & 0.758 & 0.676 & 0.597 & 0.517 & 0.437 & 0.352 & 0.264 & 0.159 & 0.094 & 0.044 & 0.022 \\
1 & 30 & 0.058 & 1.000 & 0.990 & 0.914 & 0.852 & 0.726 & 0.614 & 0.499 & 0.425 & 0.363 & 0.307 & 0.254 & 0.199 & 0.144 & 0.084 & 0.047 & 0.019 & 0.009 \\
0.1 & 30 & 0.0058 & 1.000 & 0.990 & 0.902 & 0.829 & 0.664 & 0.480 & 0.286 & 0.206 & 0.167 & 0.140 & 0.117 & 0.097 & 0.078 & 0.055 & 0.040 & 0.021 & 0.011 \\
0.01 & 30 & 0.00058 & 0.999 & 0.982 & 0.895 & 0.824 & 0.653 & 0.435 & 0.148 & 0.087 & 0.062 & 0.045 & 0.036 & 0.029 & 0.023 & 0.013 & 0.008 & 0.004 & 0.003 \\
100 & 100 & 0.25 & 1.000 & 0.995 & 0.948 & 0.909 & 0.835 & 0.760 & 0.660 & 0.579 & 0.506 & 0.437 & 0.367 & 0.294 & 0.215 & 0.124 & 0.068 & 0.029 & 0.013 \\
10 & 100 & 0.025 & 1.000 & 0.989 & 0.886 & 0.807 & 0.643 & 0.494 & 0.367 & 0.302 & 0.251 & 0.206 & 0.167 & 0.128 & 0.089 & 0.045 & 0.019 & 0.005 & 0.002 \\
1 & 100 & 0.0025 & 1.000 & 0.982 & 0.860 & 0.758 & 0.535 & 0.282 & 0.124 & 0.099 & 0.081 & 0.065 & 0.051 & 0.040 & 0.028 & 0.013 & 0.006 & 0.002 & 0.001 \\
0.1 & 100 & 0.00025 & 0.999 & 0.985 & 0.885 & 0.827 & 0.707 & 0.552 & 0.293 & 0.069 & 0.012 & 0.009 & 0.008 & 0.007 & 0.006 & 0.004 & 0.003 & 0.003 & 0.002 \\
\hline
\end{tabular}

sufficiently high for bremsstrahlung to take over. It is hydrodynamic in origin. Apart from the tail, the temperature profile at $10^{-2} \mathrm{~g} \mathrm{~cm}^{-2} \mathrm{~s}^{-1}, 100 \mathrm{MG}$ is very close to that obtained by the non-hydrodynamic approach of WB92, WB93. The low-temperature tail is responsible for a low-temperature thermal emission component with $k T \lesssim 1 \mathrm{keV}$.

The initial rise of the individual temperature profiles is similar and is very rapid following approximately $T_{\mathrm{e}} \propto x^{0.35}$ (Fig. 2). One half of $T_{\max }$ is reached at $0.001 x_{\mathrm{s}}$ in the bremsstrahlung-dominated case and at $0.006 x_{\mathrm{s}}$ near the non-hydrodynamic limit. Further downstream the profiles differ substantially. In the bremsstrahlungdominated case, the peak electron temperature is reached quickly, while in the cyclotron-dominated flow it occurs at the same $x$ at which half of the accretion energy has been radiated away. The reason is that a temperature gradient is needed to drive about one half of the radiative flux across the shock front, while the other half enters the white dwarf atmosphere. In the plane-parallel geometry, the optically thick radiative transfer requires the electron temperature at the shock front to stay below the peak 


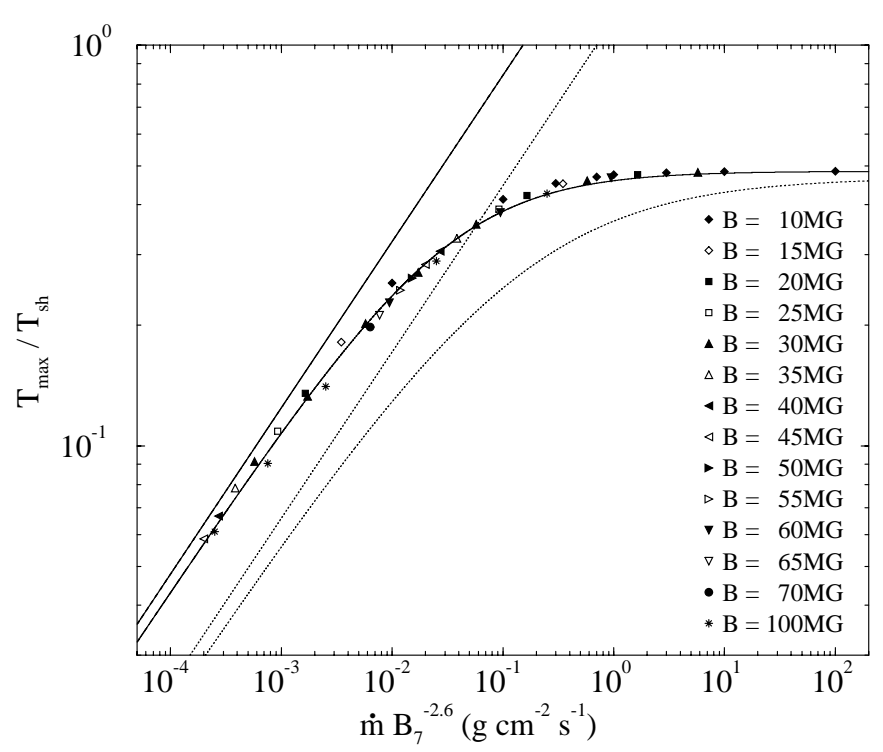

Fig. 5. Maximum electron temperature $T_{\max }$ as a function of $\dot{m} B_{7}^{-2.6}$ for $M_{\mathrm{wd}}=0.6 M_{\odot}$ and $B=10-100 \mathrm{MG}$ $\left(B_{7}=B / 10^{7} \mathrm{G}\right)$. The solid curve is the fit from Eq. (19), the solid straight line represents the bombardment solution from Eq. (18). The dotted curve and straight line represent the corresponding fit to the data for $1 M_{\odot}$ (data not shown).

electron temperature $: T_{\mathrm{e}, \mathrm{s}}<T_{\max } \leq T_{\mathrm{brems}, \max } \sim 0.5 T_{\mathrm{i}, \mathrm{s}}$. This is why we opted to start the integration with the initial values $T_{\mathrm{e}, \mathrm{s}}=0$ and $T_{\mathrm{i}, \mathrm{s}}$ as given by Eq. (7). Because of the rapid initial rise in $T(x)$, our results would have been practically the same had we set $T_{\mathrm{e}, \mathrm{s}}=0.5 T_{\max }$.

To facilitate the modeling of specific geometries, we provide the normalized temperature and density profiles for a sequence of $\dot{m}, B$ combinations in Table 1 . We also provide best fits to $T_{\max } / T_{\mathrm{i}, \mathrm{s}}$ and $x_{\mathrm{s}}$ as functions of $\dot{m} B^{-2.6}$.

\subsection{Velocity profiles for an infinite layer}

For calculations of the bremsstrahlung emission, we need the profiles of the mass density which varies as $\rho \propto v^{-1}$. Figure 4 shows the normalized velocity profiles for $M_{\mathrm{wd}}=$ $0.6 M_{\odot}$ and the same $\dot{m}, B$ combinations as in Fig. 3 . In the limit of pure bremsstrahlung cooling, the velocity profile is indistinguishable from that given by the inversion of Eq. (12). Increased cyclotron cooling causes a similar depression at intermediate $x$ as seen in the temperature profiles. Table 1 (bottom) provides the velocity profiles in numerical form for the same parameters as above.

\subsection{Maximum electron temperature $T_{\max }$}

In what follows, each model is represented by one "data point". Figure 5 shows $T_{\max } / T_{\mathrm{i}, \mathrm{s}}$ vs. $\dot{m} B_{7}^{-2.6}$ for $M_{\mathrm{wd}}=0.6 M_{\odot}$ and $B=10-100 \mathrm{MG}$. The dependence of $T_{\max }$ on $\dot{m} B_{7}^{-2.6}$ is equally well documented for $M_{\mathrm{wd}}=0.8$

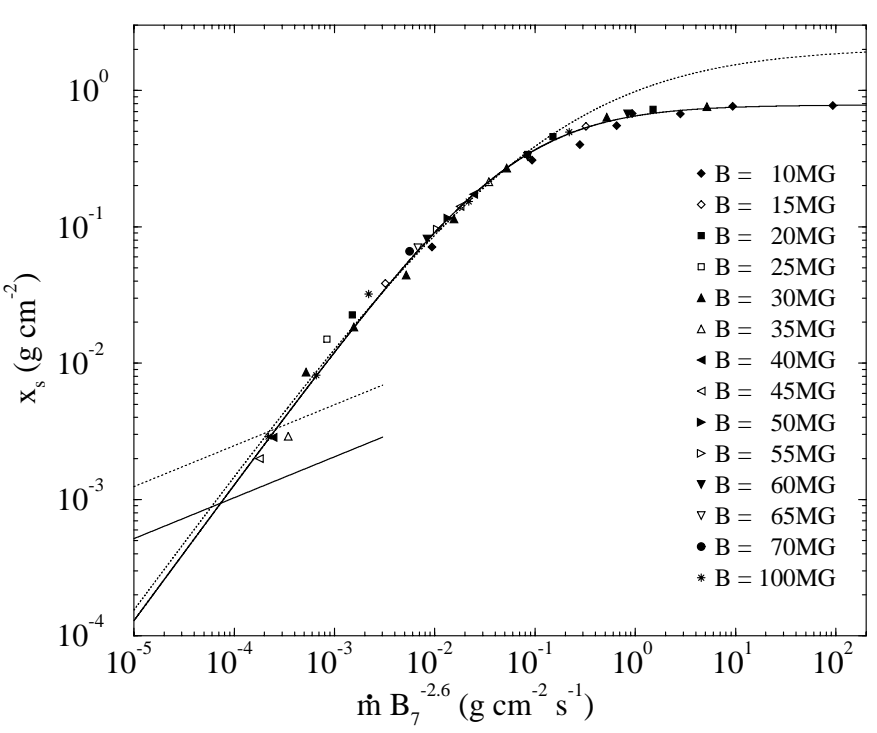

Fig. 6. Column density $x_{\mathrm{s}}$ of the post-shock cooling region as a function of $\dot{m} B_{7}^{-2.6}$ for the same parameters as in Fig. 5. The solid curve is the best fit from Eq. (20). The dotted curve indicates the corresponding fit to the data for $1 M_{\odot}$. The straight lines in the lower left denote the bombardment solutions from Eq. (17) for $0.6 M_{\odot}$ and $1.0 M_{\odot}$.

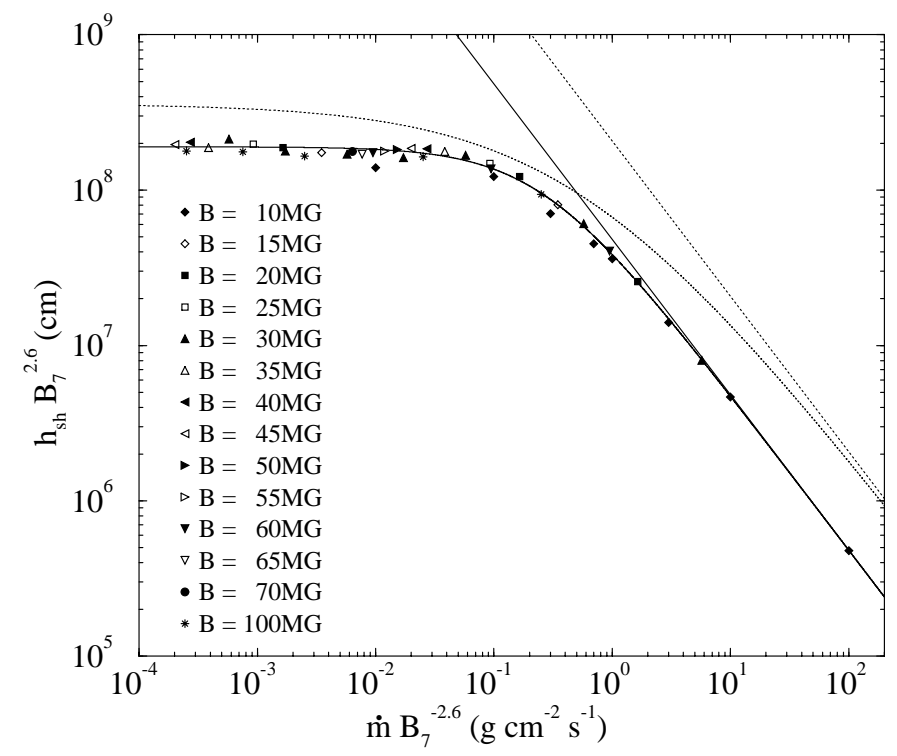

Fig. 7. Same as Fig. 6 but for geometrical shock height $h_{\mathrm{sh}}$ contained in the quantity $h_{\mathrm{sh}} B_{7}^{2.6}$. The fits refer to $0.6 M_{\odot}$ (solid curve) and $1 M_{\odot}$ (dashed curve). The straight lines represent the corresponding bremsstrahlung solutions of Eq. (14).

and 1.0 $M_{\odot}$, but for clarity we do not show these data. The $0.6 M_{\odot}$ results can be fitted by

$$
\frac{1}{T_{\max }}=\left[\left(\frac{1}{a_{0} T_{\max , \text { bomb }}}\right)^{\alpha}+\left(\frac{1}{a_{1} T_{\max , \text { brems }}}\right)^{\alpha}\right]^{1 / \alpha}
$$

with $T_{\max , \text { bomb }}$ from Eq. (18) and $T_{\max , \text { brems }}$ from Eq. (16). The exponent $\alpha$ measures the smoothness of the transition between cyclotron and bremsstrahlung solutions. The fits for 0.6 and $1 M_{\odot}$ are included in Fig. 5 as the solid and dotted curves, respectively. The fit 


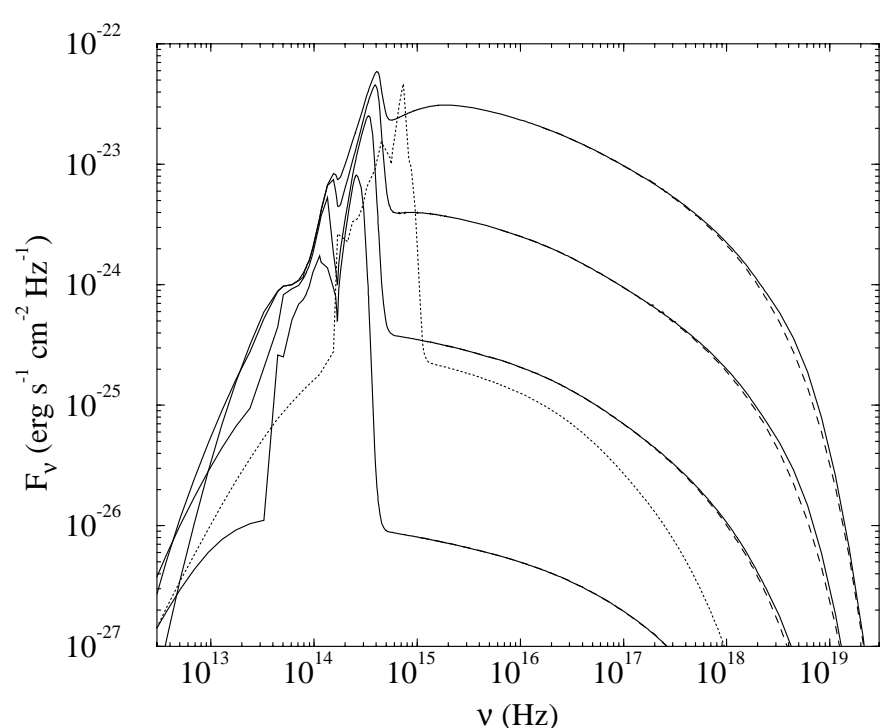

Fig. 8. Overall spectral energy distributions for an emission region on an $0.6 M_{\odot}$ white dwarf at $d=10 \mathrm{pc}$ with $B=$ $30 \mathrm{MG}, D=10^{8} \mathrm{~cm}$, and $\dot{m}=100,10,1$, and $10^{-1} \mathrm{~g} \mathrm{~cm}^{-2} \mathrm{~s}^{-1}$ (from top). The dashed sections indicate the emission directed away from the white dwarf at $\vartheta=5^{\circ}$ without the reflection albedo, the solid curves include the latter. The dotted curve for $\dot{m}=1 \mathrm{~g} \mathrm{~cm}^{-2} \mathrm{~s}^{-1}, B=100 \mathrm{MG}$ indicates the increased cyclotron radiation and the reduced bremsstrahlung flux and temperature for this field strength.

Table 2. Fit parameters of Eqs. (19)-(21) for three values of the white dwarf mass $M_{\mathrm{wd}}$.

\begin{tabular}{cccccccc}
\hline $\begin{array}{c}M \\
\left(M_{\odot}\right)\end{array}$ & $a_{0}$ & $a_{1}$ & $\alpha$ & $\begin{array}{c}b_{0} \\
(\mathrm{~s})\end{array}$ & $\beta$ & $\begin{array}{c}c_{0} \\
\left(10^{8} \mathrm{~cm}\right)\end{array}$ & $\gamma$ \\
\hline 0.6 & 0.91 & 0.968 & 1.67 & 6.5 & 0.70 & 0.95 & 1.0 \\
0.8 & 0.86 & 0.954 & 1.54 & 7.5 & 0.54 & 1.30 & 0.7 \\
1.0 & 0.90 & 0.934 & 1.25 & 8.0 & 0.45 & 1.75 & 0.5 \\
\hline
\end{tabular}

parameters $a_{0}, a_{1}$, and $\alpha$ are listed in Table 2 for all three white dwarf masses. The fact that $a_{1}$ falls slightly short of 1.0 indicates that the limiting value $T_{\max }=\left(\mu / \mu_{\mathrm{i}}\right) T_{\mathrm{i}, \mathrm{s}}$ for large $\dot{m}$ is not yet reached at $100 \mathrm{~g} \mathrm{~cm}^{-2} \mathrm{~s}^{-1}$. Even at this high $\dot{m}$, radiative energy losses remove some energy prior to equipartition. The maximum temperatures at low $\dot{m}$ remain about $10 \%$ below the temperatures predicted by the bombardment solution of WB92,93 (straight lines, $a_{0} \simeq 0.9$ ). A difference as small as this is actually remarkable considering the substantially different theoretical and numerical approaches (radiation hydrodynamics vs. radiative transfer in a static atmosphere).

\subsection{Column density $x_{\mathrm{s}}$}

The transition of $x_{\mathrm{s}}$ between the bombardment and the bremsstrahlung solutions (Eqs. (17) and (13)) is more complicated than that of $T_{\max }$. Figure 6 shows $x_{\mathrm{s}}$ as a function of $\dot{m} B_{7}^{-2.6}$ for $M_{\mathrm{wd}}=0.6 M_{\odot}$ and $B=10-100 \mathrm{MG}$. Again, the data points for 0.8 and

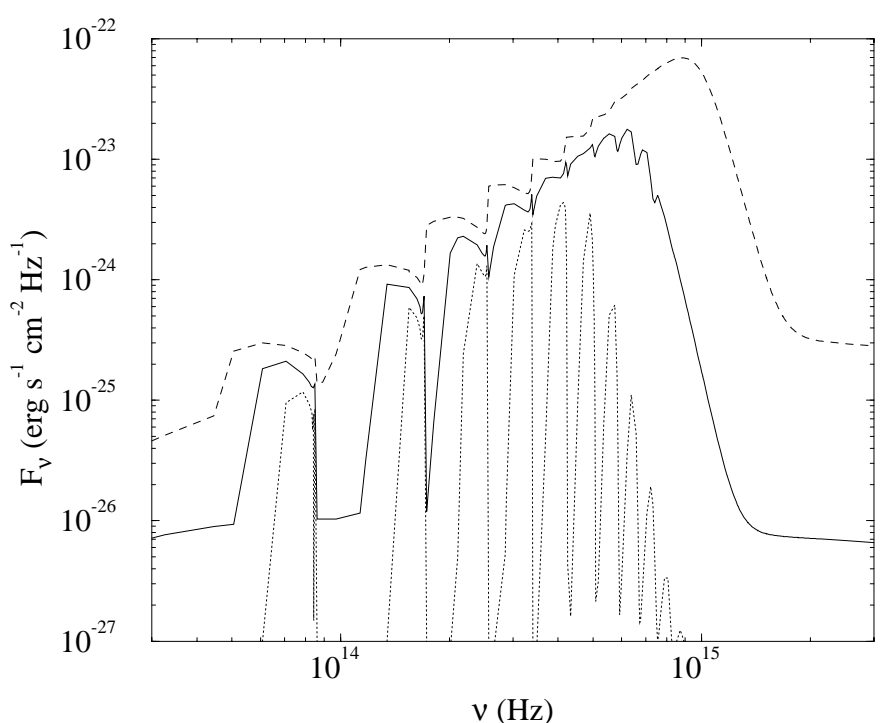

Fig. 9. Cyclotron section of the spectral energy distributions for the same set of parameters as in Fig. 8, except $\vartheta=80^{\circ}$, and for mass flow densities $\dot{m}=1,10^{-1}$, and $10^{-2} \mathrm{~g} \mathrm{~cm}^{-2} \mathrm{~s}^{-1}$ (from top).

$1.0 M_{\odot}$ are not shown for clarity. We fit $x_{\mathrm{s}}$ by

$\frac{1}{x_{\mathrm{s}}}=\left[\left(\frac{\mu}{b_{0} \mu_{\mathrm{e}} \dot{m} B_{7}^{-2.6}}\right)^{\beta}+\left(\frac{1}{x_{\mathrm{s}, \text { brems }}}\right)^{\beta}\right]^{1 / \beta}$

with $x_{\mathrm{s}, \text { brems }}$ from Eq. (13). The fit parameters $b_{0}$ and $\beta$ are given in Table 2 for the three values of $M_{\mathrm{wd}}$. Again, the fits are shown for 0.6 and $1.0 M_{\odot}$ (solid and dotted curve), with the corresponding bombardment solutions added as straight lines. Note that, contrary to what we found for $T_{\max }$, the first term in Eq. (20) does not represent the bombardment solution, but rather the cyclotrondominated shock heated plasma. It connects to the bombardment solution as the non-hydrodynamic regime is approached and bridges a gap of two orders of magnitude in $x_{\mathrm{s}}$ between the bombardment and bremsstrahlung solutions. Clearly, the quantitative determination of $x_{\mathrm{s}}(\dot{m}, B)$ requires radiation-hydrodynamical calculations. The $\dot{m}$ dependence of Eq. (20) reproduces that of Eq. (10), the molecular weight dependence is added here and taken from Eq. (9).

\subsection{Geometrical shock height $h_{\mathrm{sh}}$}

Figure 7 shows the quantity $h_{\mathrm{sh}} B_{7}^{2.6}$ for $M_{\mathrm{wd}}=0.6 M_{\odot}$ and for field strengths between 10 and $100 \mathrm{MG}$. We fit the data by

$\frac{1}{h_{\mathrm{sh}} B_{7}^{2.6}}=\left[\left(\frac{\mu}{c_{0} \mu_{\mathrm{e}}}\right)^{\gamma}+\left(\frac{B_{7}^{-2.6}}{h_{\mathrm{sh}, \text { brems }}}\right)^{\gamma}\right]^{1 / \gamma}$

where $\dot{m}$ as independent variable enters via $h_{\mathrm{sh}, \text { brems }}$ from Eq. (14) and the fit parameters $c_{0}$ and $\gamma$ are listed in Table 2. The fits for $0.6 M_{\odot}$ and $1.0 M_{\odot}$ are 
shown (solid and dotted curve). The limiting dependencies for large and small $\dot{m} B^{-2.6}$, respectively, are $h_{\mathrm{sh}}=$ $h_{\mathrm{sh}, \text { brems }} \propto \dot{m}^{-1}$ and $h_{\mathrm{sh}}=c_{0} B_{7}^{-2.6}=$ const., as predicted by Eq. (11). The shock height is related to $x_{\mathrm{s}}$ by the mean post-shock density $\bar{\rho}=x_{\mathrm{s}} / h_{\mathrm{sh}}$ which is $\bar{\rho}=6.97 \rho_{0}$ for the bremsstrahlung-dominated shock solution (see Eqs. (13) and (14)). For the cyclotron-dominated shock-heated flow, the mean post-shock density increases to $\bar{\rho}=\left(b_{0} / c_{0}\right) \rho_{0} v_{\mathrm{o}} \simeq 30 \rho_{0}$, a result which cannot be obtained from simple theory.

\section{Emission regions of finite lateral width}

In our radiation-hydrodynamical calculations, energy conservation is enforced and the radiative luminosity of the infinite layer per unit area equals the accretion energy $\dot{m} v_{\mathrm{o}}$. Any real emision region, however, has a finite lateral width and looses energy not only from its top and bottom surfaces but also from its sides (Fig. 1). Two-dimensional radiation hydrodynamics would then be needed to calculate the temperature structure. In this section we discuss to what extent our one-dimensional results are still applicable to regions of finite extent.

\subsection{Emitted spectra}

We consider an emission region as depicted in Fig. 1, with finite width $D$, cross section $D^{2}$, and field strength $B$. In a first step, we adopt the temperature and velocity profiles along the flow lines, $T(x)$ and $v(x)$, calculated for infinite $D$ also for the case of finite $D$.

In the Rybicki code the radiation transport equation was solved with a mean cyclotron absorption coefficient and electron scattering was included. For the emission region of finite extent, we calculate the outgoing flux at angle $\vartheta$ by ray tracing using the temperature profiles along slanted paths as shown schematically in Fig. 1. For rays starting or ending on the side surfaces, the temperature and density profiles were truncated appropriately. We account separately for the cyclotron emissivities in the ordinary ray (index o) and the extraordinary ray (index e), and add $50 \%$ of the free-free emissivity with Gaunt factor to both. We neglect electron scattering in the emission region, but include the atmospheric albedo $A_{\nu}$ (van Teeseling et al. 1994). Each ray yields a contribution $\Delta \mathcal{I}_{\nu}$ to the integrated intensity $\mathcal{I}_{\nu}$ (in $\mathrm{erg} \mathrm{s}^{-1} \mathrm{~Hz}^{-1} \mathrm{sr}^{-1}$ ) in that direction and the summation is extended over $n$ rays,

$$
\begin{aligned}
\mathcal{I}_{\nu}(\vartheta)= & \sum_{i=1}^{n} \Delta \mathcal{I}_{\nu}(\vartheta)=\sum_{i=1}^{n} \Delta \sigma^{(i)}\left(\int_{0}^{s_{\text {max }}^{(i)}} \epsilon_{\nu, \mathrm{o}}^{(i)}(\vartheta, s) \mathrm{e}^{-\tau_{\nu, \mathrm{o}}^{(i)}(\vartheta, s)} \mathrm{d} s\right. \\
& \left.+\int_{0}^{s_{\max }^{(i)}} \epsilon_{\nu, \mathrm{e}}^{(i)}(\vartheta, s) \mathrm{e}^{-\tau_{\nu, \mathrm{e}}^{(i)}(\vartheta, s)} \mathrm{d} s\right)
\end{aligned}
$$

where $\tau_{\nu, \mathrm{o}}^{(i)}(\vartheta, s)$ and $\tau_{\nu, \mathrm{e}}^{(i)}(\vartheta, s)$ are the optical depths of the ordinary and extraordinary rays along path $i$ at angle $\vartheta, s_{\max }^{(i)}$ is the pathlength along that ray and $\Delta \sigma^{(i)}$ the effective projected area associated with it. The albedo contribution at $\vartheta$ is calculated as $A_{\nu} \mathcal{I}_{\nu}(\pi-\vartheta)$ and is not yet included in Eq. (22). Reprocessing of the flux absorbed in the white dwarf atmosphere is not considered in this paper and the corresponding flux is, therefore, missing from our spectra. The spectral luminosity $L_{\nu}$ is obtained by integrating Eq. (22) over $4 \pi$ and the total luminosity $L$ by integration over all frequencies.

Figure 8 shows the spectral flux at $\vartheta=5^{\circ}$ against the field direction emitted by an emission region with $B=30 \mathrm{MG}$ and an area of $10^{16} \mathrm{~cm}^{2}\left(D=10^{8} \mathrm{~cm}\right)$ on an $0.6 M_{\odot}$ white dwarf at a distance of $10 \mathrm{pc}$. Cyclotron emission dominates for low $\dot{m}$ and bremsstrahlung for high $\dot{m}$. Free-free absorption becomes important near $10^{15} \mathrm{~Hz}$ at the highest $\dot{m}$, but in reality this spectral region is dominated by the quasi-blackbody component produced by reprocessing of the incident flux in the white dwarf atmosphere. The results of WB96 on the ratio of the cyclotron vs. bremsstrahlung luminosities as a function of $\dot{m}$ and $B$ remain basically valid, but will be modified if the shock is buried in the atmosphere and X-ray absorption is accounted for.

Figure 9 illustrates the optical depth dependence of the cyclotron spectra at $\vartheta=80^{\circ}$. Cyclotron emission lines at low $\dot{m}$ change into absorption features at high $\dot{m}$. Since in real emission regions the fractional area of the high- $\dot{m}$ section is small (Rousseau et al. 1996) observed spectra show emission lines.

\subsection{Specific luminosity}

An isolated emission region of lateral width $D$, shock height $h_{\mathrm{sh}}$, and the temperature profile $T(x)$ of the infinite layer appropriate for the mass flow density $\dot{m}$ will have $\mathcal{L}=\dot{m} v_{\mathrm{o}}$ for optically thin and $\mathcal{L} \geq \dot{m} v_{\mathrm{o}}$ for optically thick emission. The overestimate in the latter case results from radiation emerging from the sides of the region without a compensating influx. For the optical depths considered here, bremsstrahlung is practically free of such overestimate, cyclotron radiation is not.

Let us assume for the infinite layer that $\dot{m} v_{\mathrm{o}}$ feeds two components of $\mathcal{L}$, namely $\mathcal{L}_{\text {thin }}$ and $\mathcal{L}_{\text {thick }}=\dot{m} v_{\mathrm{o}}-\mathcal{L}_{\text {thin }}$. For finite $D$, we then have

$\mathcal{L} \simeq \mathcal{L}_{\text {thin }}+\left(\dot{m} v_{\mathrm{o}}-\mathcal{L}_{\text {thin }}\right)\left(1+\frac{2 h_{\mathrm{sh}}}{D}\right) \geq \dot{m} v_{\mathrm{o}}$

where the second term in brackets is the ratio of the total surface area of the emission region over the sum of top and bottom areas. For simplicity, we have neglected the temperature variation over the surface of the emission region and taken the energy loss per unit area as constant. We rearrange the terms in Eq. (23) to form a quantity $A$ which relates $\mathcal{L} / \dot{m} v_{\text {o }}$ to the aspect ratio of the emission region, $h_{\mathrm{sh}} / D$,

$$
A=\left(\frac{\mathcal{L}}{\dot{m} v_{\mathrm{o}}}-1\right) \frac{D}{2 h_{\mathrm{sh}}} \simeq 1-\frac{\mathcal{L}_{\text {thin }}}{\dot{m} v_{\mathrm{o}}}
$$




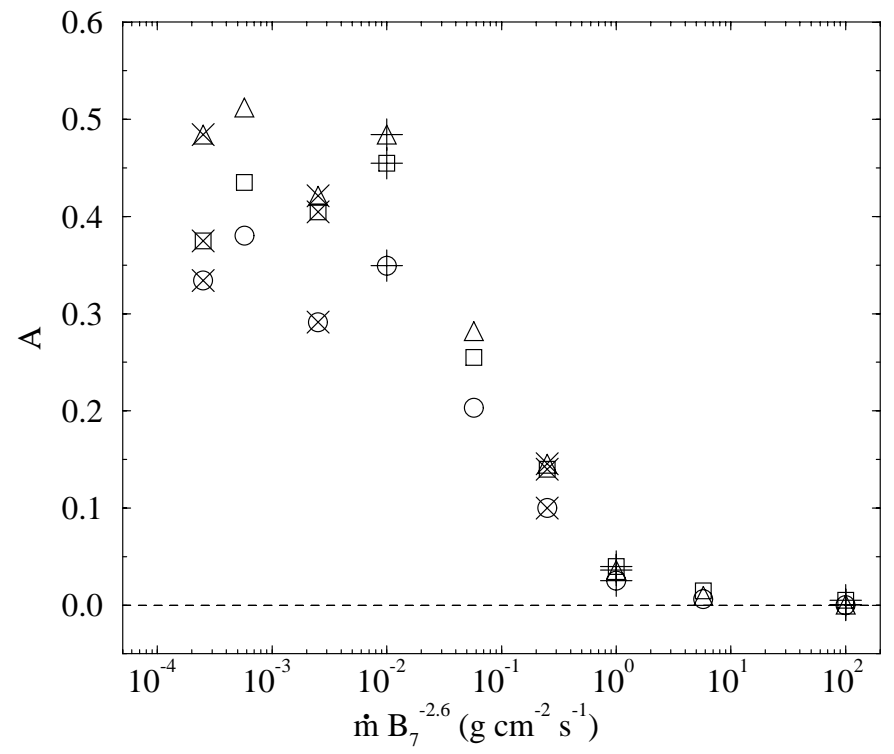

Fig. 10. Quantity $A$ from Eq. (24) measuring the excess luminosity $\mathcal{L} / \dot{m} v_{\text {o }}$ as function of the aspect ratio of the emission region, $h_{\mathrm{sh}} / D$. The symbols refer to narrow and pillbox-shaped emission regions with $D / h_{\mathrm{sh}}=0.1(\bigcirc), D / h_{\mathrm{sh}}=1(\square), D / h_{\mathrm{sh}}=$ $10(\triangle)$, the overplotted + and $\times$ refer to field strengths of 10 and $100 \mathrm{MG}$, respectively, the uncrossed symbols to $B=$ $30 \mathrm{MG}$.

Figure 10 shows $A$ as a function of $\dot{m} B_{7}^{-2.6}$, calculated for model columns with $h_{\mathrm{sh}} / D=0.1,1$ and 10 in the way described in the previous section. To a first approximation, $A$ is independent of $h_{\mathrm{sh}} / D$ and the relative luminosity error $(\mathcal{L}-\dot{m}) / \dot{m} v_{\text {o }}$ increases proportional to $h_{\mathrm{sh}} / D$ for a given $\dot{m} B_{7}^{-2.6}$. The quantity $A$ is negligibly small for large $\dot{m} B_{7}^{-2.6}$ where bremsstrahlung dominates and reaches $A \simeq 0.4$ for low $\dot{m} B_{7}^{-2.6}$ where cyclotron radiation dominates. Note that $A$ never reaches the optically thick limit of unity because bremsstrahlung and optically thin cyclotron emission always contribute. To give an example, $h_{\text {sh }} / D=0.5$ and $A \simeq 0.4$ imply $\mathcal{L} / \dot{m} v_{\mathrm{o}} \simeq 1.4$, i.e. an overestimate of the luminosity by $40 \%$.

In order to assess the size of the possible error for AM Her stars, we estimate $h_{\mathrm{sh}} / D$ for a typical accretion rate of $\dot{M}=10^{16} \mathrm{~g} \mathrm{~s}^{-1}$ as a function of $\dot{m}$. The linear width of the emission region is $D \simeq(\dot{M} / \dot{m})^{1 / 2} \simeq 10^{8} / \dot{m}^{1 / 2} \mathrm{~cm}$ with $\dot{m}$ in $\mathrm{g} \mathrm{cm}^{-2} \mathrm{~s}^{-1}$. For a bremsstrahlung-dominated flow, Eq. (14) yields $h_{\text {sh, brems }} / D \simeq 0.48 / \dot{m}^{1 / 2}$ (for $M_{\mathrm{wd}}=$ $\left.0.6 M_{\odot}\right)$ which is less than unity since in this case $\dot{m} \gtrsim$ $1 \mathrm{~g} \mathrm{~cm}^{-2} \mathrm{~s}^{-1}$. For a cyclotron-dominated flow, Eq. (21) correspondingly yields $h_{\text {sh,cyc }} / D \simeq 2 \dot{m}^{1 / 2} / B_{7}^{2.6}$ which is again less than unity since now $\dot{m} \lesssim 1 \mathrm{~g} \mathrm{~cm}^{-2} \mathrm{~s}^{-1}$ and typically $B_{7}>1$. Hence, $\mathcal{L}$ is seriously overestimated only for isolated narrow subcolumns which are not radiatively shielded by neighboring fluxtubes.

\subsection{Temperature correction}

Let the application of the unmodified one-dimensional temperature profile $T(x)$ yield a specific luminosity
$\mathcal{L}=r \cdot \dot{m} v_{\text {o }}$ with $r>1$. We can then take then either: (i) identify the parameters of this emission region with those appropriate for the increased mass flow rate $\dot{m}^{\prime}=$ $r \cdot \dot{m}$; or (ii) recalculate the emission for a reduced mass flow rate $\dot{m}^{\prime \prime}=\dot{m} / r$ and identify temperature and emission of that region as appropriate for the initial $\dot{m}$. In case (ii), $T_{\max }$ and $x_{\mathrm{s}}$ are reduced to $T_{\max }\left(\dot{m}^{\prime \prime}\right)$ and $x_{\mathrm{s}}\left(\dot{m}^{\prime \prime}\right)$. This approach demonstrates that the rising sections of the relations displayed in Fig. 5 (Eq. (19)) and Fig. 6 (Eq. (20)) are further depressed for narrow columns, while the horizontal parts, where optically thin bremsstrahlung dominates, are not affected. Both approaches secure energy conservation but cannot replace a proper treatment of the problem. They are not recommended for isolated tall columns.

\section{Discussion}

We have solved the equations of one-dimensional, twofluid stationary radiation hydrodynamics for the shockheated plasma in the emission regions on accreting magnetic white dwarfs for a wide range of mass flow densities $\dot{m}=\rho_{0} v_{\mathrm{o}}$ and field strengths $B$. For given $B$ and $M_{\mathrm{wd}}$, the peak electron temperature $T_{\max }$ and the column density $x_{\mathrm{s}}$ of the emission region are physically related to $\dot{m}$ as the independent variable of the theory. They are no longer independent variables as in the frequently employed "constant- $\Lambda$ models". It is possible, therefore, to interpret the observed spectral energy distributions of accreting magnetic white dwarfs in terms of the distribution of mass flow densities present in their accretion spots.

We now discuss to what extent the application of these results is limited by the simplifications made in our calculations. One major simplification is the assumption of stationarity which implies that we neglect the possible occurrence of shock oscillations (Imamura et al. 1996; Saxton \& $\mathrm{Wu} 1999$, and references therein) and that we can not treat rapid time variability of $\dot{m}$. Since our approach can accommodate a range of $\dot{m}$ to occur in neighboring columns, the emitted spectrum will still approximate the true timeaveraged spectrum if $\dot{m}$ varies only on time scales exceeding the post-shock cooling time $\tau_{\text {cool }} \simeq 4 h_{\text {sh }} / v_{\mathrm{o}} \sim 1 \mathrm{~s}$. In the presence of shock oscillations which have periods of order $\tau_{\text {cool }}$, our results yield a mean temperature and column density which need not agree with the true timeaveraged value if the oscillation is nonlinear (Imamura \& Wolff 1990).

The assumption of a one-dimensional flow implies that we neglect the convergence of the polar field lines of the white dwarf. In the spirit of this approximation, we have included the acceleration of the post-shock flow by a constant gravity $g=\mathrm{G} M_{\mathrm{wd}} / R_{\mathrm{wd}}^{2}$ and neglected the $r$ dependence of $g$.

The assumption of an infinite layer implies that there is no temperature gradient perpendicular to the flow. This is no restriction for bremsstrahlung-dominated flows, but in such gradient is always established in columns of finite width $D$ by optically thick radiation components and 
lowers the mean electron temperature averaged across the column at any position $x$. We have suggested a simple first-order correction for the implied overestimate in $T(x)$ which ensures conservation of energy and provides some remedy for narrow columns with $h_{\mathrm{sh}} \sim D$. For very narrow columns with $h_{\mathrm{sh}} \gg D$ or absolutely tall columns with $h_{\mathrm{sh}}>0.1 R_{\mathrm{wd}}$, the main radiative energy flow may be sideways and the approach of Wu et al. (1994) becomes more appropriate. In summary, our results are valid whenever $h_{\text {sh }} \ll R_{\text {wd }}$ and $h_{\text {sh }} \lesssim D$.

On the positive side, we consider our largely correct treatment of the two-fluid nature of the post-shock flow. One-fluid treatments (e.g. Chevalier \& Imamura 1982; Wu et al. 1994) can account for cooling by cyclotron radiation in addition to bremsstrahlung, but are limited, by definition, to mass flow densities sufficiently high to ensure quick equilibration of electron and ion temperatures. They can not describe the substantial reduction of the peak electron temperature below the one-fluid value which we show to be present at low mass-flow density $\dot{m}$ and/or high magnetic field strength $B$. As a result, our description catches the essential properties of such columns: (i) dominant cyclotron cooling causes the peak electron temperature to stay far below the peak temperature of the one-fluid approach; (ii) cyclotron cooling causes a drastic reduction in the column density and the geometrical shock height of the post-shock flow compared with pure bremsstrahlung cooling; and (iii) peak temperature and column density vary smoothly between the two limiting cases, the bremsstrahlung-dominated high- $\dot{m}$ regime (Aizu 1973) and the cyclotron-dominated low- $\dot{m}$ bombardment solution (WB92, WB93). The latter denotes the transition to the non-hydrodynamic regime and, gratifyingly, our calculations recover the bombardment solution at the lowest mass flow densities accessible. Compared with WB96, we obtained numerically more accurate results and have cast these into simple-to-use fit formulae which facilitate the modeling of emission regions within the geometrical limitations noted above. No other twofluid calculations with the full optically thick radiative transfer are available.

The remaining, mainly geometrical limitations of our approach are inherently connected to the one-dimensional radiative transfer. Extension of the calculation to two dimensions encounters two problems: (i) a substantial increase in complexity and (ii) the introduction of an additional free parameter in form of the lateral width of the emission region. Therefore, we consider our one-dimensional approach with the correction explained in Sect. 3 as a reasonable compromise, with the noted exception of tall columns.
The present results can be used to quantitatively model the emission regions on accreting magnetic white dwarfs. The discussion of the overall spectral energy distribution of such objects requires to account for shocks being buried in the photosphere of the white dwarf. Such model allows to obtain the $\dot{m}$-distribution in the accretion spot from observational data and is presented in Paper II of this series. The emission properties of AM Herculis binaries depend not only on $\dot{m}$ but vary also systematically with field strength: this dependence is described in Paper III.

Acknowledgements. This work is based on a code originally devised by U. Woelk. We thank B. T. Gänsicke, F. V. Hessman and K. Reinsch for numerous discussions and the referee J. Imamura for helpful comments which improved the presentation of the results. This work was supported in part by BMBF/DLR grant 50 OR 99036.

\section{References}

Aizu, K. 1973, Prog. Theor. Phys., 49, 1184

Chanmugam, G., \& Dulk, G. A. 1981, ApJ, 244, 569

Chanmugam, G., \& Langer, S. H. 1991, ApJ, 368, 580

Chevalier, R. A., \& Imamura, J. N. 1982, ApJ, 261, 543

Imamura, J. N., Durisen, R. H., Lamb, D. Q., \& Weast, G. J. 1987, ApJ, 313, 298

Imamura, J. N., \& Wolff, M. T. 1990, ApJ, 355, 216

Imamura, J. N., Aboasha, A., Wolff, M. T., \& Wood, K. 1996, ApJ, 458, 327

Imamura, J. N., Steiman-Cameron, T. Y., \& Wolff, M. T. 2000, PASP, 112, 18

Kuijpers, J., \& Pringle, J. E. 1982, A\&A, 114, L4

Lamb, D. Q., \& Masters, A. R. 1979, ApJ, 234, L117

Larsson, 1992, A\&A, 265, 133

Nauenberg, M. 1972, ApJ, 175, 417

Rousseau, T., Fischer, A., Beuermann, K., \& Woelk, U. 1996, A\&A, 310, 526

Saxton, C. J., \& Wu, K. 1999, MNRAS, 310, 677

Spitzer, L. 1956, Physics of fully ionized gases (Interscience Publ. Inc.)

Thompson, A. M., \& Cawthorne, T. V. 1987, MNRAS, 224, 425

van Teeseling, A., Heise, J., \& Paerels, F. 1994, A\&A, 281, 119

Wada, T., Shimizu, A., Suzuki, M., Kato, M., \& Hoshi, R. 1980, Prog. Theor. Phys., 64, 1986

Woelk, U., \& Beuermann, K. 1992, A\&A, 256, 498 (WB92)

Woelk, U., \& Beuermann, K. 1993, A\&A, 280, 169 (WB93)

Woelk, U., \& Beuermann, K. 1996, A\&A, 306, 232 (WB96)

Wolff, M. T., Wood, K. S., Imamura, J. N., Middleditch, J., \& Steiman-Cameron, T. Y. 1999, ApJ, 526, 453

Wu, K., Chanmugam, G., \& Shaviv, G. 1994, ApJ, 426, 664 